\title{
Editorial: Pre-registration and Assessing Effects of Commonly Used Techniques in Animal Behavior Research
}

\author{
Michael J. Beran ${ }^{1}$ \\ ${ }^{1}$ Georgia State University \\ Corresponding author (Email: mberan1@gsu.edu) \\ Citation - Beran, M. J. (2020). Pre-registration and assessing effects of commonly used techniques in animal behavior \\ research. Animal Behavior and Cognition, 7(4), 490-491. doi: https://doi.org/10.26451/abc.07.04.01.2020
}

Keywords - Pre-registration, Methodology, Animal behavior

Animal Behavior and Cognition offers authors the opportunity to engage in peer-reviewed feedback of pre-registered research projects (Vonk \& Krause, 2018). The process involves submitting a manuscript that is much like a grant proposal in that the rationale and justification for a research project are outlined prior to data collection. The specific methods are described, the data to be collected are outlined, and the planned analyses of those data are indicated. The implications of possible outcomes of the research project also are described, and from this, the peer reviewers can determine the significance of the research question and the feasibility and appropriateness of the methodological approach and data analysis plan. Reviewers then indicate their opinions on the value of doing the work, but without any sense of how the data will turn out.

This submission type is an essential part of anticipating and addressing the replication crisis in social science (Nosek \& Lakens, 2014). Peer-reviewed pre-registrations are an excellent tool for avoiding bias on the part of authors and reviewers. On the review side, there is no concern that the outcome of the research leads to harsh reviews that intentionally or unintentionally reflect a reviewer's bias against the possibility the new data may challenge older data or older theories and ideas. In other words, turf wars are avoided. In addition, peer-reviewed pre-registration avoids the possibility that authors and reviewers cannot agree on what data and how much data are needed after the data collection phase has ended. In animal behavior research, this is really important because it is often impossible to collect more data as animals are no longer accessible for a variety of reasons. At the same time, peer-reviewed pre-registration anticipates and resolves any disagreement as to how to analyze data before those data are collected. Without this resolution, there are increased chances of possible "stats-hunting" to find statistically significant (or non-significant) outcomes before concluding data collection. Pre-registration in general (with or without peer review) ensures that research teams make clear what they intended to do before data collection began, thereby minimizing the risk of p-hacking (Stevens, 2017) and HARKing (Kerr, 1998), as can occur when there is no contract with oneself at the outset of a study as to how you will conduct that study. For these reasons, and others, Animal Behavior and Cognition embraces this submission format.

In this issue, we are pleased to publish one such peer-reviewed, pre-registered report. This paper (Tysall et al., 2020) specifically focused on an assessment of commonly used methods to mark flies in behavioral research. The pre-registration indicated the need to determine whether marking methods of flies that were then studied in mating contexts could, in fact, affect such mating behavior. The process worked as designed, with reviewers aiding the research team to design a method and analysis plan that all parties agreed would allow for the best way to answer this question. With this completed, the outcome was considered by all parties to be important and worthy of publication, no matter what that specific outcome was. As you can read, a comparison of five marking methods commonly used for Drosophila melanogaster 
produced some interesting outcomes, and there could be important implications for those studying mating behavior in that species.

People studying animal behavior often use methods that are considered by the field to be appropriate because they do not change or influence the behavior of interest simply as a result of their use, but that may not always be true. Assessing how true this is for commonly used methods is an excellent opportunity to engage in peer-reviewed pre-registration and also serve an important role in our field for increasing confidence in our results (Koole \& Lakens, 2012), and we encourage research teams to consider the peerreviewed pre-registration format for their own studies that could benefit from its strengths.

\section{References}

Kerr, N. (1998). HARKing: Hypothesizing after the results are known. Personality and Social Psychology Review, 2, 196-217.

Koole, S. L., \& Lakens, D. (2012). Rewarding replications: A sure and simple way to improve psychological science. Perspectives on Psychological Science, 7, 608-614.

Nosek, B. A., \& Lakens, D. D. (2014). Registered reports: A method to increase the credibility of published results. Social Psychology, 45, 137-141.

Stevens, J. R. (2017). Replicability and reproducibility in comparative psychology. Frontiers in Psychology, 8, 862.

Tysall, E. E., Pembury Smith, M. Q. R., \& Gilman, R. T. (2020). The effects of marking methodology on mate choice in Drosophila melanogaster. Animal Behavior and Cognition, 7, 492-504.

Vonk, J. \& Krause, M. A. (2018). Editorial: Announcing preregistered reports. Animal Behavior and Cognition, 5, iii. 